\title{
Fibromatosis of the cecum presenting with acute appendicitis: a case report
}

This article was published in the following Dove Press journal:

International Journal of General Medicine

2I December 20II

Number of times this article has been viewed

\author{
Toygar Toydemir' \\ Gökhan Ertuğrul ${ }^{2}$ \\ 'Istanbul Surgery Hospital, \\ Department of General Surgery, \\ Nisantasi-Istanbul, ${ }^{2}$ Atatürk State \\ Hospital, Department of General \\ Surgery, Düzce, Turkey
}

Correspondence: Toygar Toydemir General Surgery Department, Istanbul Surgery Hospital,

Nisantasi-Istanbul 34365, Turkey

Tel +905053743368

Fax +90 212 2969473

Email toygartoydemir@hotmail.com

\begin{abstract}
Although acute appendicitis is a common clinical condition in general surgical practice, $<1 \%$ of them are associated with malignancies. Appendiceal carcinoids make up most of those malignancies and acute appendicitis cases associated with benign cecal neoplasias are very uncommon. In this study, a 25 -year-old female patient who presented with distinct acute appendicitis symptoms is reported. The patient was operated on via open technique. Exploration revealed an appendix with advanced edema and hyperemia. While the cecum was observed to be normal, a solid mass of $2.5 \mathrm{~cm}$ diameter was palpated in the appendiceal base. Following the ileocecal resection, histopathological examination revealed the mass as a fibromatosis. The goals of this report are to remind health care professionals that some very rare etiologies may be involved in acute appendicitis diagnosis and treatment, and to underscore the place of laparoscopic approach and preoperative computed tomography in this disease.
\end{abstract}

Keywords: acute appendicitis, fibromatosis, laparoscopy, preoperative computed tomography

\section{Introduction}

Intra-abdominal fibromatosis are uncommon benign neoplasms which originate from musculoaponeurotic structures of the body and primarily affects the mesentery or retroperitoneum. However, rarely, they can arise from the intestinal wall and mimic a gastrointestinal malign neoplasm. ${ }^{1}$ Acute appendicitis is a common clinical condition, however, $<1 \%$ of patients with acute appendicitis have an accompanying malignant process. $^{2}$ Acute appendicitis cases associated with benign lesions in the cecum are very rare. This is a report of a 25 -year-old female patient with a cecal fibromatosis causing acute appendicitis. The purpose of this report is to remind health care practitioners that some very rare etiologies may be involved in a common clinical condition and to underscore the place of laparoscopic approach and preoperative computed tomography in this disease.

\section{Case report}

A 25-year-old female patient presented to our emergency department because of abdominal pain, nausea, and vomiting within the last 24 hours. The pain started as a diffuse abdominal pain and eventually localized in the right lower quadrant. Physical examination revealed muscular rigidity and rebound tenderness in the right lower quadrant. She had fever $\left(38.5^{\circ} \mathrm{C}\right), 90$ beats per minute heart rate, and $110 / 70$ blood pressure. She had no history of surgical operations, alcohol consumption, or smoking. Laboratory analysis revealed 17,000 leukocytes with a prevalence of neutrophils. Other laboratory results were normal. Gynecological examination showed no pathological findings. Ultrasonography demonstrated a noncompressed tubular lesion in the right lower quadrant which was 
reported to be consistent with acute appendicitis. The patient was operated on with the prediagnosis of acute appendicitis. Laparotomy was performed via McBurney incision. Exploration revealed an appendix with advanced edema and hyperemia. While the cecum was observed to be normal, a solid mass of $2.5 \mathrm{~cm}$ diameter was palpated in the appendiceal base. Intraabdominal exploration showed no distinct lymphadenopathy or palpable mass in the liver. The mass was obstructing the appendix lumen and cecum resection was decided (Figure 1). Ileocecal resection and end-to-side anastomosis were performed. Postoperative pathological examination demonstrated spindle cells in myxoid stroma (Figure 2). Immunohistochemically, the lesion was diffuse strong positive with vimentin and actin, and negative for desmin, CD34, CD117, and S-100, which was reported to be a fibromatosis of $2.5 \mathrm{~cm}$ diameter. No postoperative complication developed and the patient was discharged on the fifth day. Control endoscopy performed 2-months postoperatively showed no pathology. The 2-year follow-up of the patient exhibited no recurrence.

\section{Discussion}

Fibromatosis, also known as desmoid tumors, are local aggressive benign neoplasms which originate from musculoaponeurotic structures of the body. They may have a peripheral or intra-abdominal localization. Fibromatosis may be associated with Gardner's syndrome and familial polyposis coli. ${ }^{3}$ Colonoscopy can show the colonic polyps whereas fundoscopy can display multiple pigmented lesions in the retina. While intra-abdominal fibromatosis generally originates from the mesentery of the small intestine, there are also involvements reported in the retroperitoneum, transverse mesocolon, and omentum. ${ }^{4}$ Rarely, it can also stem from the intestinal wall like submucosal benign lesions such as inflammatory fibroid polyp, leimyoma, etc, and gastrointestinal stromal tumor (GIST), and mimic a gastrointestinal malign neoplasia. Pathology bears importance in differential

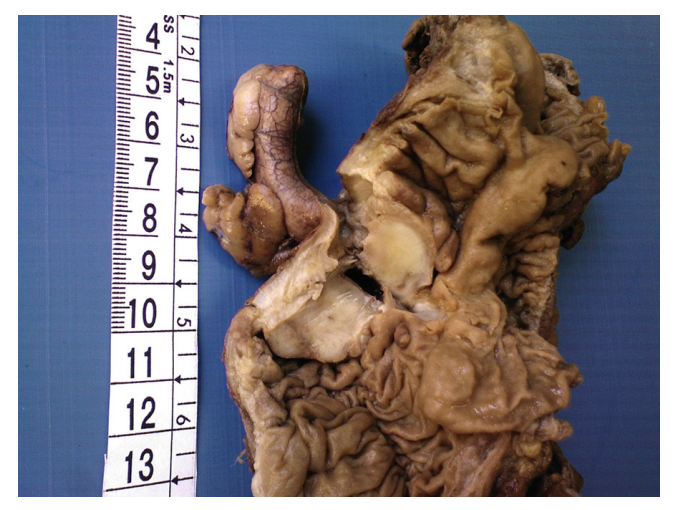

Figure I Fibromatosis occluding the lumen of appendix.

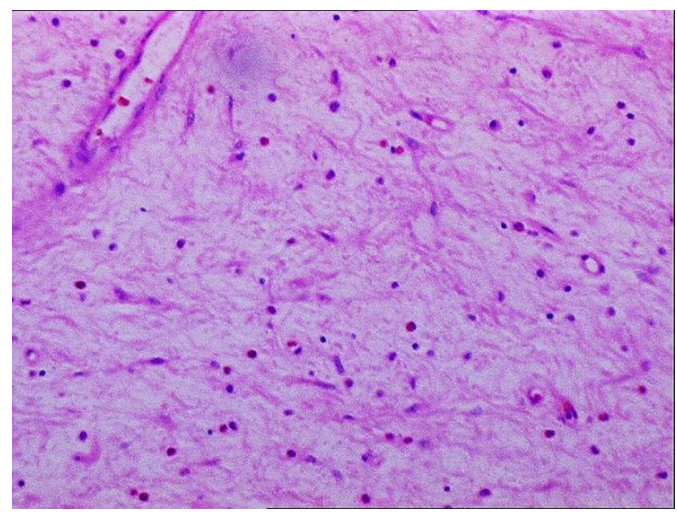

Figure 2 The lesion consists of spindle cells in myxoid stroma containing prominent vasculature and inflammatory cells.

diagnosis. GISTs are the most common mesenchymal tumor of the gastrointestinal tract and the treatment strategy is important because of its malignant potential. As a result, surgeons give special attention to GISTs and a specimen with this suspected diagnosis is important to pathologists. Absence of reaction with CD117 and S-100 is the key point of differential diagnosis between fibromatosis and GIST as in the present case.

Intra-abdominal fibromatosis are local aggressive neoplasias which do not show metastatic tendency. While most cases have an asymptomatic course, urgent surgical interventions have been reported due to reasons such as intestinal obstruction, perforation, and abscess formation. ${ }^{5}$

In women and the elderly, establishing an acute appendicitis diagnosis is problematic. The appendix has been found to be normal among $32 \%-45 \%$ of appendectomies performed on women between 15 and 45 years of age. ${ }^{6}$ Pelvic inflammatory disease, graft follicle rupture, ectopic pregnancy rupture, and ovarian cyst pathologies may be hard to differentiate from acute appendicitis. Less than $1 \%$ of patients with acute appendicitis have an accompanying malignant process, most commonly appendiceal carcinoids. Less frequently, acute appendicitis may be the initial symptom of a cecal malignancy and acute appendicitis cases associated with benign lesions in the cecum are very rare. There are very few studies that have been reported about cecal fibromatosis. A search of PubMed ${ }^{7}$ with the keywords "intra-abdominal fibromatosis," "desmoid tumour," and "cecal fibromatosis," found only one case about a cecal fibromatosis causing appendicitis ${ }^{8}$ and this report seems to be the first case in the English language.

Since the advent of video-assisted technology in general surgery, many operations have been performed by laparoscopy. Laparoscopic approach has several obvious advantages over open surgery such as less postoperative pain, reduced duration of hospitalization and incision-site infection, and achievement of a better cosmetic appearance. ${ }^{9}$ Many health care centers 
perform laparoscopic appendectomy routinely. We, as well, have applied this approach for the past 5 years.

Although the laparoscopic approach has significant advantages over open surgery, since the advent of the technique the most important criticism about it has been lack of tactile sensation by palpation. Excessive fat, adhesions occurring due to earlier surgeries, and lack of tactile sensation may cause some lesions to go unnoticed.

Shayani ${ }^{10}$ performed colonoscopy on a patient due to detection of mucin islets on the appendiceal base by histopathological examination after the laparoscopic appendectomy. A submucosal mass was seen in the cecum during the colonoscopy and the patient was subjected to right hemicholectomy via laparotomy. In the histopathological examination, the mass was found to be a cecal carcinoid. However, the lesion in the cecum was not detected in the first intervention.

In the current case, we applied open appendectomy. The cecum appeared to be totally normal and ileocecal resection was decided due to detection of a mass only by palpation. If this intervention had been performed laparoscopically, this lesion would probably not have been detected.

However, the application of a preoperative computed tomography (CT) could have revealed the lesion of $2.5 \mathrm{~cm}$ diameter prior to the procedure. Multislice CT benefits detection of ileocecal region diseases such as mesentery lymphadenopathy, ischemic colitis, cecal tumors, Crohn's disease, and, particularly, acute appendicitis. ${ }^{11}$ Clinical evaluation of right lower quadrant pain is carried out with scorings (Alvarado ${ }^{12}$ ) and most of the surgeons resort to CT imaging in cases with low scores or suspected symptoms. Since our patient had high scores, we deemed preoperative CT evaluation unnecessary.

A recently published meta-analysis study reported that the negative appendectomy rate is $8.7 \%$ when using CT compared with $16.7 \%$ when using clinical evaluation alone $(P<0.001) .{ }^{13}$ Although routine use of CT in all patients presenting with suspected appendicitis may lead to delay in surgery, this delay is not associated with increased appendiceal perforation of morbidity rates. ${ }^{10}$ However, it is important to know whether routine use of CT is cost-effective in patients with suspected right lower quadrant pain. Morse and colleagues demonstrated

International Journal of General Medicine

\section{Publish your work in this journal}

The International Journal of General Medicine is an international, peer-reviewed open-access journal that focuses on general and internal medicine, pathogenesis, epidemiology, diagnosis, monitoring and treatment protocols. The journal is characterized by the rapid reporting of reviews, original research and clinical studies across all disease areas. that multislice CT reduces the negative appendectomy rates and savings averaged US\$1412 per patient. ${ }^{14}$

In conclusion, surgeons should be aware that some very rare etiologies may be involved in acute appendicitis management. Cecal fibromatosis is one of the least common reasons for acute appendicitis and preoperative diagnosis is difficult. CT can be helpful in both detection of unpredicted coexisting lesions and reduction of negative appendectomy.

\section{Disclosure}

The authors report no conflict of interest in this work.

\section{References}

1. Yantiss RK, Spiro IJ, Compton CC, Rosenberg AE. Gastrointestinal stromal tumor versus intra-abdominal fibromatosis of the bowel wall. A clinically important differential diagnosis. Am J Surg Pathol. 2000; 24(7):94-957.

2. Blair NP, Bugis SP, Turner LJ, MacLeod MM. Review of the pathologic diagnoses of 2,216 appendectomy specimens. Am J Surg. 1993;165: 618-620.

3. Vaswani BA, Shah M, Shah PM, Parikh BJ, Anand AS, Sharma GL. Giant mesenteric fibromatosis in Gardner's syndrome. Indian J Cancer. 2011;48:140-142.

4. Gonatas NK. Extra-abdominal desmoid tumors. Report of six cases. Arch Pathol. 1961;71:214-221.

5. Jalini N, Hemming D, Bhattacharya V. Intraabdominal desmoid tumour presenting with perforation. Surgeon. 2006;4(2):114-116.

6. Flum DR, Koepsell T. The clinical and economic correlates of misdiagnosed appendicitis: nationwide analysis. Arch Surg. 2002;137:799-804

7. PubMed.gov [database on the Internet]. Bethesda, MD: National Center for Biotechnology Information, US Library of Medicine; nd. Available from: http://www.ncbi.nlm.nih.gov/pubmed/. Accessed December 19, 2011.

8. Kurguzov OP, Sergeev VV. Acute appendicitis in a patient with fibroma of the cecum. Khirurgiia (Mosk). 1995;(5):72-73. Russian.

9. Hoeffel JC, Weryha B, Ravey M, Prenat T, Kurdziel JC. Fibroma of the cecum. Case report. Diagn Imaging Clin Med. 1986;55(6):376-380.

10. Shayani V. Mucinous cystadenoma of the cecum missed at laparoscopic appendectomy. Pitfalls in laparoscopy. Surg Endosc. 1999;13(12): 1236-1237.

11. Hoeffel C, Crema MD, Belkacem A, et al. Multi-detector row CT: spectrum of diseases involving the ileocecal area. Radiographics. 2006; 26(5):1373-1390.

12. McKay R, Shepherd J. Alvarado in the decision to perform computed tomography for acute appendicitis in the ED. Am J Emerg Med. 2007;25(5):489-493.

13. Krajewski S, Brown J, Phang PT, Raval M, Brown CJ. Impact of computed tomography of the abdomen on clinical outcomes in patients with acute right lower quadrant pain: a meta-analysis. Can J Surg. 2011;54(1):43-53.

14. Morse BC, Roettger RH, Kalbaugh CA, Blackhurst DW, Hines WB Jr. Abdominal CT scanning in reproductive-age women with right lower quadrant abdominal pain: does its use reduce negative appendectomy rates and healthcare costs? Am Surg. 2007;73(6):580-584.

\section{Dovepress}

A key focus is the elucidation of disease processes and management protocols resulting in improved outcomes for the patient.The manuscript management system is completely online and includes a very quick and fair peer-review system. Visit http://www.dovepress.com/ testimonials.php to read real quotes from published authors. 